\title{
Wachsende Stadtregion - Modellrechnungen zum Bevölkerungswachstum in der Stadtregion OST
}

\author{
Heinz FASSMAnN und Peter GöRgL, beide Wien* \\ mit 5 Abb. und 2 Tab. im Text
}

\begin{tabular}{|c|c|}
\hline \multicolumn{2}{|r|}{ INHALT } \\
\hline & $\ldots .183$ \\
\hline & 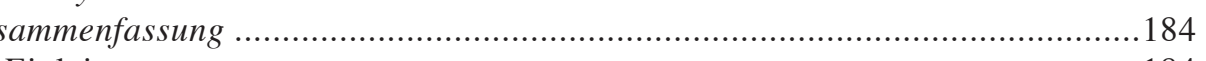 \\
\hline & 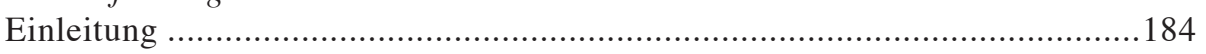 \\
\hline & 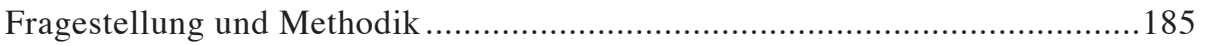 \\
\hline & Fortschreibung der Trends: Status-Quo-Szenario \\
\hline & Bürgermeisterszenario \\
\hline & Interventionsszenarien \\
\hline & Fazit ................................ \\
\hline & Literaturverzeichnis ....................... \\
\hline
\end{tabular}

\section{Summary}

A Growing Urban Region - Model Calculations on Population Growth in the Urban Region East

The Urban Region East, the Vienna Region, will grow further. When in the year 2009 around 2.6 million people were living in the agglomeration (1.7 million in Vienna [Wien] and ca.900,000 in its surroundings), we will face a number of around 3 million in 2030 ( 2 millions in the city of Vienna and 1 million in the surroundings). This lets for the first time in history the question arise, how this growth can be managed. By model calculations this question was answered under various assumptions: Which spatial structure emerges, when current trends are extrapolated into the future? How will growth spread over the urban region, when the wishes of communal representa-

* Univ.-Prof. Dr. Heinz FASSMANN, Institut für Geographie und Regionalforschung, Universität Wien, Universitätsstraße 7, A-1010 Wien; E-mail: heinz.fassmann@univie.ac.at, http://www.univie.ac.at/ geographie; Dr. Peter GöRGL, Institut für Stadt- und Regionalforschung, Österreichischen Akademie der Wissenschaften, Postgasse 7/4/2,A-1010 Wien; E-mail: peter.goergl@oeaw.ac.at, http://www.oeaw.ac.at/isr 
tives are respected? And how many people is the urban region able to host, when growth is confined to parts of the urban region, which are easily accessible? This is the topic of this contribution.

\section{Zusammenfassung}

Die Stadtregion Ost, die Vienna Region, wird weiter wachsen. Lebten im Jahr 2009 rund 2,6 Mio. Menschen in der Agglomeration (1,7 Mio. in Wien und rund $900.000 \mathrm{im}$ Umland), so werden es 2030 rund 3 Mio. sein (2 Mio. in der Stadt Wien und 1 Mio. im Umland). Es erhebt sich daher historisch erstmals die Frage, wie dieses neue Wachstum bewältigt werden kann. Im Rahmen von Modellrechnungen wurde diese Frage unter unterschiedlichen Prämissen beantwortet: Welche räumliche Struktur stellt sich ein, wenn Trends fortgeschrieben werden? Wie verteilt sich das Wachstum in der Stadtregion, wenn es nach den Vorstellungen der Kommunalverantwortlichen geht? Und wie viele Menschen kann die Stadtregion aufnehmen, wenn die Aufnahme auf die gut erreichbaren Teile der Stadtregion beschränkt bleibt? Darüber berichtet der folgende Beitrag.

\section{Einleitung}

Das wissenschaftliche Interesse am Stadtumland als einer von der Kernstadt abhängigen, aber zunehmend auch selbständigen Siedlungseinheit hat in den vergangenen Jahren zugenommen. Auslöser war dabei eine mehrheitlich kritische Sichtweise auf die ökologischen und sozialen Folgen einer zunehmenden Verlagerung von Wohnbevölkerung und Arbeitsplätzen aus der Kernstadt in das Stadtumland. Der Begriff des „Speckgürtels“, der in vielen, auch wissenschaftlichen Publikationen verwendet wurde, brachte und bringt die mentale Reservation sehr deutlich zum Ausdruck.

Eine Abkehr von der normativen Festlegung der Stadtumlandentwicklung als etwas „Schlechtem“, das auch planerisch zu verhindern sei, gelang dem Ladenburg-Kolleg, welches sich dieses Themas annahm, mehrere Jahre aktiv war, Forschung initiierte und einschlägig publizierte und dabei den weitaus neutraleren Begriff der Zwischenstadt verwendete (vgl. Bölling \& SieverTs 2004, BraKe et al. 2005). Analog dazu rückte auch die Wiener Stadtregion in der jüngeren Vergangenheit immer mehr in den Fokus unterschiedlich konzipierter Forschungsarbeiten und Analysen. Die Bandbreite reicht dabei von der Analyse grundsätzlicher funktionaler Stadt-Umland-Beziehungen auf der Makroebene (LoiBL et al. 2002) oder der Modellierung von suburbanen Entwicklungsprozessen (HeLbich 2009) hin zu Analysen spezifischer Teilräume der Stadtregion (z.B. der Wienerwaldgemeinden in MusiL \& PINDUR 2008) oder konkreter Siedlungsprojekte (z.B. des Wohnparks „Fontana“ in GörgL 2008).

Der vorliegende Beitrag stellt eine konkrete Frage in den Mittelpunkt: Wie aufnahmefähig ist das Stadtumland von Wien? Wie viele zusätzliche Einwohner können 
unter Zugrundelegung unterschiedlicher Siedlungsparameter im Stadtumland untergebracht werden ohne zusätzliches Bauland aufzuschließen? Es geht in diesem Beitrag nicht um die Analyse bisher zu beobachtender Suburbanisierungsprozesse, auch nicht um die Abschätzung der ökologischen, wirtschaftlichen oder sozialen Folgen der Suburbanisierung und schon gar nicht um den Entwurf theoretischer Konzepte, die zur Begründung der aktuellen Situation herangezogen werden könnten. Die hier vorgestellte Forschungsfrage ist begrenzt, dafür aber eindeutig und politisch ausgesprochen relevant, denn sie führt zu einer klaren Schlussfolgerung. Die kommunale Autonomie im Bereich der Flächenwidmung und Entwicklungskonzeption muss durch übergeordnete Planungskonzepte und Planungsinstitutionen eingerahmt und damit auch begrenzt werden, um zukünftige Wachstumsprozesse bewältigen zu können.

\section{Fragestellung und Methodik}

Wie wird die Stadtregion Ost (Vienna Region) ihr prognostiziertes Wachstum bewältigen, ohne dass die Nachhaltigkeit auf der Strecke bleibt? Dazu werden im Folgenden Szenarien der räumlichen Entwicklung der Stadtregion entworfen und berechnet, um abschätzen zu können, was die Folgen einer sich fortsetzenden Suburbanisierung sein könnten. Diese Szenarien sind mehr als grobe Gedankenskizzen, die nur unscharf möglicherweise Kommendes antizipieren. Sie sind vielmehr Modellrechnungen, die konkret und präzise die Folgen eines bestimmten Sets von Annahmen berechnen.

Dazu wurden drei unterschiedliche Szenarien und Ausgangskonstellationen entworfen. Das erste Szenario lautet: Fortschreibung der bisherigen Wachstumstrends. Dieses Status-Quo-Szenario zielt darauf ab, die Auswirkungen eines fortgeschriebenen Wachstums auf das verfügbare Bauland und die stadtregionale Verteilung zu überprüfen. In welchen Gemeinden nimmt die Wohnbevölkerung zu? Stoßen diese Gemeinden an ihre durch das gewidmete Bauland vorgegebenen Wachstumsgrenzen? Und entspricht das räumliche Muster des Wachstums den planerischen Vorstellungen? Bei den Status-Quo-Szenarien werden die gemeindespezifischen Wachstumsraten der Jahre 2004-2008 fortgeschrieben. Des Weiteren wird einmal davon ausgegangen, dass $100 \%$ der Baulandreserve und ein anderes Mal 50\% mobilisierbar sind. Und es wird damit gerechnet, dass es zu keiner Nachverdichtung kommt oder diese nur in einem sehr geringen Ausmaß stattfindet. Räumliche Bezugs- und Recheneinheit ist die Gemeinde (vgl. Tab. 1).

Das zweite Szenario stützt sich auf die Angaben der kommunalpolitisch Verantwortlichen. Aus diesem Grund wird das Szenario auch „Bürgermeisterszenario“ genannt, obwohl die Bürgermeister nicht immer unmittelbare Gesprächspartner waren. Wachstumsraten werden in diesem Szenario jedenfalls nicht linear fortgeschrieben, sondern basieren auf den Wünschen und Absichten der befragten Personen. „Was wollen die Gemeindeverantwortlichen?", lautet die Grundfrage dieses Szenarios. Das Gleiche gilt für die Mobilisierung des Baulandes. Dabei wird auf eine Befragung der kommunalpolitisch Verantwortlichen in den 268 Umlandgemeinden zurückgegriffen und 


\begin{tabular}{|l|l|l|l|}
\hline Annahmen & $\begin{array}{l}\text { Status-quo-Szenarien } \\
\text { (SQ1 und SQ2) }\end{array}$ & Bürgermeisterszenario & Interventionsszenario \\
\hline $\begin{array}{l}\text { Bevölkerungs- } \\
\text { entwicklung }\end{array}$ & $\begin{array}{l}\text { bis 2030 konvergier- } \\
\text { te Wachstumsraten } \\
\text { 2004-2008 (auf 1,1\% } \\
\text { p.a.) }\end{array}$ & $\begin{array}{l}\text { Wachstum durch An- } \\
\text { gaben der Kommunal- } \\
\text { politik vorgegeben }\end{array}$ & $\begin{array}{l}\text { Bevölkerungsentwick- } \\
\text { lung Ergebnis des Auf- } \\
\text { füllens von Bauland }\end{array}$ \\
\hline $\begin{array}{l}\text { Mobilisierung } \\
\text { von Bauland }\end{array}$ & $\begin{array}{l}\text { SQ1: vollständige } \\
\text { Mobilisierung; } \\
\text { SQ2: Hälfte wird } \\
\text { mobilisiert }\end{array}$ & $\begin{array}{l}\text { empirische Erhebung; } \\
\text { bei fehlenden Angaben: } \\
\text { Verwendung des Durch- } \\
\text { schnitts }\end{array}$ & $\begin{array}{l}\text { variabel (60\% in Zellen } \\
\text { mit Hochpotenzial, } \\
\text { 40\% mit Mittelpotenzial } \\
\text { und 20\% mit Niedrig- } \\
\text { potenzial) }\end{array}$ \\
\hline Verdichtung & $\begin{array}{l}\text { SQ1: keine Verdich- } \\
\text { tung; } \\
\text { SQ2: Erhöhung um } \\
+10 \%\end{array}$ & $\begin{array}{l}\text { empirische Erhebung; } \\
\text { bei fehlenden Angaben: } \\
\text { Verwendung des Durch- } \\
\text { schnitts }\end{array}$ & $\begin{array}{l}\text { variabel (50 Einw./ha in } \\
\text { den Zellen mit Hoch- } \\
\text { potenzial, 40 Einw./ha } \\
\text { im Mittelpotenzial und } \\
\text { 30 Einw./ha im Nied- } \\
\text { rigpotenzial; zusätzlich } \\
\text { 10\% in den Kernen der } \\
\text { Zentralen Orte) }\end{array}$ \\
\hline $\begin{array}{l}\text { Räumliche } \\
\text { Bezugsebene }\end{array}$ & Gemeinde & Gemeinde & \begin{tabular}{l} 
Raster \\
\hline
\end{tabular}
\end{tabular}

Tab. 1: Definition der Szenarien

deren Einschätzung dem Szenario zugrunde gelegt. ${ }^{1)}$ Dort, wo keine Angaben getätigt wurden, kommt die Annahme einer mittleren Baulandmobilisierung zur Anwendung. Ähnlich wird bei der Nachverdichtung verfahren. Wenn Angaben über die geplante Nachverdichtung vorliegen, dann werden diese für das Szenario verwendet. Im Falle von fehlenden Angaben wird ein Mittelwert eingesetzt. Die Berechnungen beziehen sich abermals auf die Gemeinden.

Das dritte Szenario wird Interventionsszenario genannt und der Titel signalisiert den Inhalt. Es geht nicht um das Fortschreiben gegenwärtiger Trends oder um die Umsetzung der Wünsche und Erwartungen der kommunalpolitisch Verantwortlichen, sondern um ein Auffüllen der vorhandenen Siedlungsflächen unter Zugrundelegung bestimmter Besiedlungsparameter. Wachstum soll dort erfolgen, wo dies aufgrund planerischer Prämissen sinnvoll ist und es soll in einer Art und Weise stattfinden, die den suburbanen Charakter der Besiedlung nicht grundsätzlich verändert. Wie viele Menschen können untergebracht werden, wenn man das vorhandene und auch verfügbare Bauland in den gut ausgestatteten und gut erreichbaren Räumen für Wohnzwecke nützt und dabei bestimmte Dichtewerte annimmt? Ist es möglich, den vorhergesagten Zuwachs an Wohnbevölkerung dort unterzubringen, wo sich die Räume dafür auch eignen? Wenn das der Fall wäre, dann ist ein wesentliches Resultat erbracht: Wachstum

\footnotetext{
1) Das Projektseminar „Die Zukunft der Wiener Suburbia“ wurde an der Universität Wien im SS 2009 durchgeführt und von Elisabeth GRUBER organisatorisch betreut. Sie war auch dafür verantwortlich, dass der Erhebungsplan eingehalten wurde und die umfangreichen Recherchearbeiten in geordneten Bahnen verliefen.
} 
kann stattfinden und es wird eine ökologisch nachhaltige Entwicklung der Stadtregion nicht gefährden. Es müssen dann ,nur“ noch die Instrumente gefunden werden, um die zusätzliche Wohnbevölkerung auch tatsächlich dorthin zu lenken, wo man sie haben will. Das ist - das wissen alle Experten - immer noch eine große Aufgabe, aber die Erreichbarkeit dieses Ziels ist nachgewiesen.

Im Interventionsszenario sind also die Wachstumsraten der Bevölkerung nicht Input, sondern Ergebnis eines planerisch legitimierten Besiedlungsprozesses. Dabei werden die suburbanen Räume aufgrund der vorhandenen Ausstattungsqualität und Erreichbarkeit in Hochpotenzial-, Mittelpotenzial- und Niedrigpotenzialgebiete eingeteilt. In den Hochpotenzialgebieten werden $60 \%$ des vorhandenen Baulandes mobilisiert, in den Mittelpotenzialgebieten $40 \%$ und in den Niedrigpotenzialgebieten 20\%. Ähnlich differenziert erfolgt die Nachverdichtung. In den Hochpotenzialgebieten wird eine Erhöhung der Bebauungsdichte auf 50 Einwohner pro ha angestrebt, in den Mittelpotenzialgebieten auf 40 Einwohner pro ha und in den Niedrigpotenzialgebieten auf 30 Einwohner pro ha. Zusätzlich wird eine Erhöhung der Bebauungsdichte im Bestand der Zentralen Orte um 10\% vermutet und auch planerisch angestrebt. Räumliche Einheiten der Berechnungen sind nicht mehr die Gemeinden, sondern Rasterzellen mit einer Rasterweite von 1,5 km. Das gesamte Untersuchungsgebiet umfasst 3.600 Rasterzellen.

Die Abgrenzung der Stadtregion mit 268 Gemeinden entspricht derjenigen, die im Rahmen des Forschungsprojekts „Strategien zur Entwicklung der Ostregion (SRO)“ der Planungsgemeinschaft Ost (PGO) im Jahr 2008 festgelegt wurde (vgl. Atlas der wachsenden Stadtregion, FASSMANN et al.2010). Dabei handelt es sich um eine relativ großzügige Abgrenzung, die von den Planungspraktikern der drei Länder Wien, Niederösterreich und Burgenland vorgenommen wurde. Es handelt sich dabei, aber das muss hier nicht betont werden, um keine Region mit eigenen politisch-planerischen Kompetenzen, sondern nur um eine statistische Zusammenführung von Gemeinden, die mit der Kernstadt Wien in einem raumfunktionellen Zusammenhang stehen.

\section{Fortschreibung der Trends: Status-Quo-Szenario}

Die Status-Quo-Szenarien basieren auf der Fortschreibung der Wachstumstrends der Jahre 2004 bis 2008. ${ }^{2)}$ In diesen Jahren betrug die durchschnittliche Wachstumsrate der 272 Umlandgemeinden (bezogen auf die mittlere Bevölkerung von 2006) im Schnitt $+1,1 \%$. Rund 240 Gemeinden wiesen ein positives Wachstum auf, $32 \mathrm{Ge}$ meinden eine Abnahme. Die höchsten Abnahmen (zwischen -1\% und -2\%) fanden sich in Klein-Neusiedl, Wallern im Burgenland, Tadten und Klingenbach. Die höchsten

\footnotetext{
2) Um den Einfluss des Zeitfensters zu relativieren und extreme Ausreißer in die eine oder andere Richtung zu vermeiden, wurden konvergierte Wachstumsraten verwendet; und zwar derart, dass die hohen Wachstumsraten verringert und die niedrigen Raten erhöht wurden. Die Annahme, die damit implizit akzeptiert wird, lautet: hohe Wachstumsraten gehen zurück, weil die entsprechenden Gemeinden ihre Wachstumsgrenzen erreicht haben. Umgekehrt bemühen sich wachstumsschwache Gemeinden verstärkt um Wohnbevölkerung. Es kommt damit zu einer Form der Umverteilung des Wachstums und zu einer langfristigen Konvergenz der Wachstumsraten.
} 
Zunahmen waren in den Gemeinden Obersiebenbrunn, Neusiedl am See, Gänserndorf, Leopoldsdorf, Steinbrunn, Parndorf, Raasdorf, Hagenbrunn, Gießhübl, Gramatneusiedl, Muckendorf-Wipfing, Mitterndorf an der Fischa sowie Haslau-Maria Ellend vorzufinden. Diese Gemeinden profitierten von verbesserter Erreichbarkeit, vergleichsweise niedrigen Bodenpreisen und auch vom deklarierten Wunsch, weiterhin Bevölkerung aufnehmen zu wollen.

Die errechneten Einwohnerzahlen der Status-Quo-Szenarien werden bei gegebener Dichte mit dem zusätzlich notwendigen Bauland und mit der verfügbaren Baulandreserve in Beziehung gesetzt. Es gilt dabei besonderes Augenmerk auf die Frage zu legen, ob das errechnete Wachstum mit den vorhandenen Baulandreserven in Einklang steht. ${ }^{3)}$ Dieser Vergleich erfordert Annahmen über die Einwohnerdichte und das Ausmaß des nicht gehorteten Baulandes, die in Tabelle 1 dargestellt sind. Die erste Variante (SQ1) hat tatsächlich den Charakter einer Modellrechnung und geht davon aus, dass $100 \%$ der gesamten Baulandreserve auch tatsächlich nutzbar sind. Es wird auch angenommen, dass das Bauland in der gleichen Dichte wie bisher genutzt wird. Die zweite Variante (SQ2) nähert sich der Frage realistischer und nimmt an, dass lediglich 50\% des Baulandes nutzbar gemacht werden können und sich die Einwohnerdichte um $10 \%$ erhöht. Ihr liegt damit die Annahme zugrunde, dass Preissteigerungen auf dem Immobilienmarkt durch ein begrenztes Angebot sowie eine steigende Nachfrage zu kleineren Grundstücken und einer erhöhten Wohnbaudichte führen.

Die Bevölkerungsentwicklung ist in beiden Status-Quo-Szenarien (SQ1 und SQ2) der relevante Ausgangsparameter. Wird sie fortgeschrieben, dann erhöht sich die Bevölkerungszahl des Stadtumlands von 909.000 im Jahr 2008 auf 1,039.000 im Jahr 2020. Diese Zunahme um rund 130.000 liegt in einer nicht unplausiblen Größenordnung, ist jedoch ausschließlich das Ergebnis einer linearen Fortschreibung der konvergierten Trends der Jahre 2004 bis 2008. Diese mögliche Zunahme liegt deutlich über der von Statistik Austria prognostizierten Zunahme der Bevölkerung in Niederösterreich um 106.000 bis zum Jahr 2020

In einem nächsten Schritt wird die zusätzliche Einwohnerzahl bei gegebener Dichte auf die Fläche umgelegt. Wie viel zusätzliches Bauland ist zur Unterbringung der Bevölkerung notwendig, und ist dieses zusätzliche Bauland als Reserve vorhanden? „Zumeist“, lautet die Antwort, denn am Ende des Betrachtungszeitraums ist die Restreserve der Gemeinden des Stadtumlands immer noch beträchtlich, Abbildung 1 belegt dies. Gemeinden, die im Jahr 2020 immer noch eine Reserve aufweisen, sind rot gekennzeichnet. Gemeinden, die über keine Reserven mehr verfügen oder diese überkonsumiert haben werden, sind blau markiert. Das Kartenbild ist vorwiegend rot und nur wenige Gemeinden in unmittelbarer Nachbarschaft zu Wien haben einen Blauton. Besonders hohe Baulandreserven (über 20\% des Restbestandes) finden sich im nördlichen Burgenland sowie im südlichen Wiener Becken (Steinfeld). Vereinzelt und mosaikartig angeordnet, was auf den isolierten Effekt der kommunalen Widmungspraxis schließen lässt, finden sich Gemeinden mit hohen Restreserven im Wienerwald, im Marchfeld sowie im Tullner Feld. Eine übergeordnete und von raumordnerischen Gesichtspunkten geleitete Verteilung ist nicht erkennbar, was sicherlich die Notwen-

\footnotetext{
3) Die Flächenangaben über Baulandnutzung und Baulandreserve wurden von den beteiligten PGOInstitutionen der Länder Wien, Niederösterreich und Burgenland bereitgestellt.
} 
digkeit andeutet, stärker stadtregional zu denken. Es ist anscheinend doch weniger die „Aufnahmekapazität" das Problem als die raumstrukturelle Anordnung des Wachstums.

Das Szenario SQ1 stellt eine Modellrechnung dar, um die Grenzen der „Aufnahmekapazität" des Stadtumlandes zu identifizieren. Dabei kann man noch weiter gehen und fragen, wie viele Einwohner mit der bestehenden Baulandreserve und bei gegebener gemeindespezifischer Dichte ihr Auslangen finden würden. Es wären zusätzlich 260.000 Einwohner, also doppelt so viele wie bei Fortschreibung des Trends bis 2021 zu erwarten sind. Erhöht man die geringe Dichte um 50\%, dann erhöht sich auch die Zahl der Neubürger, die im Stadtumland ihren Wohnort beziehen könnten, auf 390.000. Lässt man auch eine mäßige Nachverdichtung im Bestand um 25\% zu, dann erhöht sich die „Aufnahmekapazität“ auf zusätzlich 618.000 Menschen.

Szenario SQ1 belegt die große theoretisch mögliche „Aufnahmekapazität“ des Stadtumlands, im Wesentlichen hervorgerufen durch die großzügigen Baulandreserven, die in der Vergangenheit gewidmet wurden. Diese großen Baulandreserven sind aber nur theoretisch verfügbar, denn das Phänomen des Hortens ist seit vielen Jahrzehnten ein ständiger Begleiter der Baulandwidmung: Baugrund wird gehortet, weil man ihn als Geldanlage betrachtet oder eine Nutzung erst für später plant. Die Befragung der Gemeindeverantwortlichen hat auch ergeben, dass rund zwei Drittel des gesamten Baulandes eigentlich nicht verfügbar sind. Daher gelangt nur ein Teil der Baulandreserve auf den Markt und kann zur Befriedigung der Wohnwünsche verwertet werden. SQ1 bleibt also mit einer Markterschließung des Baulandes von 100\% unrealistisch.

Szenario SQ2 führt daher die Argumentation weiter. Es nimmt an, dass in der Regel nicht die gesamte Reserve auf den Markt gelangt, sondern nur ein Teil davon. Angenommen wird eine 50\%-ige Aktivierung des Baulands. Auch diese Annahme ist noch optimistisch, denn der tatsächliche Wert liegt etwa bei einem Drittel und nur im Falle einer verstärkten Nachfrage nach Bauland auch darüber.

Eine zweite Überlegung ist SQ2 noch voranzustellen: Ein hohes Bevölkerungswachstum in den Gemeinden führt in der Realität nicht zu einer Fortschreibung der bisherigen, sondern zu einer erhöhten Bebauungsdichte. Viele Zuzüge in eine Gemeinde haben - bei Konstanthaltung des Baulands - höhere Nachfrage und eine Verteuerung von Grund und Boden zur Folge. Mit der Verteuerung steigt aber auch der Druck, die Fläche intensiv zu nutzen. Im Bereich der Wohnhausverbauung heißt das: Reihenhausverbauung und mehrgeschossige Wohnhausanlagen verdrängen einzeln stehende Einfamilienhäuser, die durchschnittliche Parzellengröße nimmt ab, die Bebauungsdichte steigt. ${ }^{4)}$ Die Annahme um 20\% erhöhter Dichtewerte ist ausgesprochen vorsichtig, denn der sich daraus ergebende Durchschnittswert von rund 36 Personen pro ha ist „städtebaulich“ noch weit von einer dichten Verbauung entfernt. Er entspricht immer noch einer Einzelhausverbauung.

Was sich im Szenario SQ2 nicht verändert, ist die Bevölkerungszahl im Jahr 2021. Es wurden weiterhin die Wachstumsraten der Jahre 2004 bis 2008, abgemildert um die Konvergenzannahme, angewendet. Auch nach SQ2 wird im Jahr 2021 das Stadtumland

\footnotetext{
4) Ein Blick in die Daten belegt, dass das auch gut möglich ist. Der Median der Dichtewerte in den 268 Gemeinden des Stadtumlandes liegt bei 26,3 Einwohnern pro ha der verbauten Siedlungsfläche (nur sonstiges Bauland), das arithmetische Mittel bei rund 30, was einer ausgesprochen lockeren Verbauung entspricht. 30 Einwohner pro ha heißt auch, dass drei Personen in einer Wohnung bzw. einem Gebäude leben, welches auf einer großzügigen Parzelle von $1.000 \mathrm{~m}^{2}$ errichtet wurde.
} 


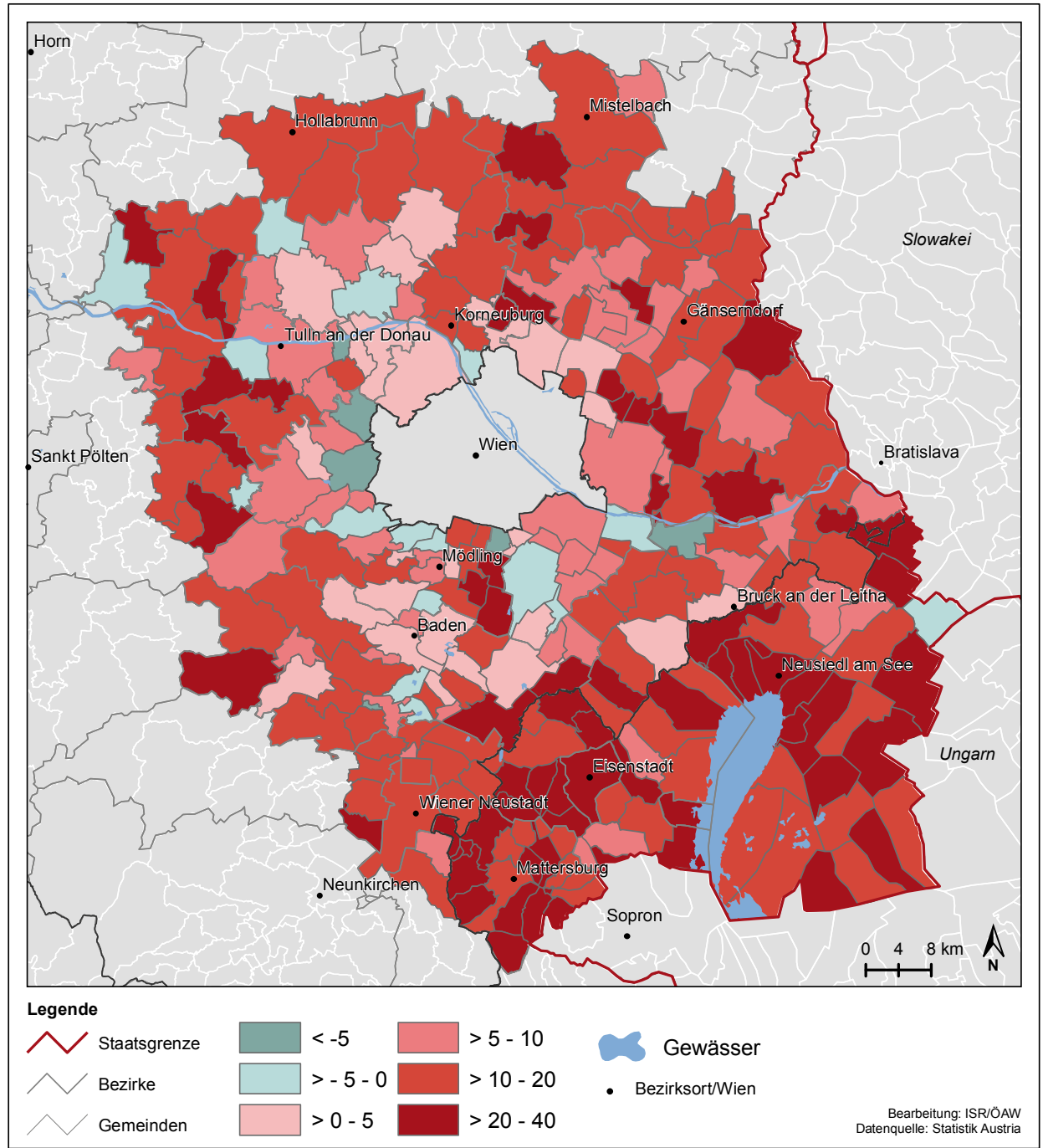

Abb. 1: Restreserve Bauland 2020 (Status-Quo-Szenario 1)

zusätzlich rund 130.000 Einwohner beherbergen. Die Verarbeitung dieses Wachstumsdrucks ist in SQ2 jedoch drängender als in SQ1, denn es wird ja angenommen, dass nur noch die Hälfte der Reserve verfügbar ist und die Verdichtung diesen Ausfall nur teilweise kompensieren kann. Die Folgen dieser Annahmen sind daher deutlich: Nicht mehr bloß 22 Gemeinden stoßen an ihre Wachstumsgrenzen, sondern bereits in über 70 Gemeinden liegt der Bedarf an Bauland über der (marktfähigen) Baulandreserve. Einem Viertel der Umlandgemeinden geht das verfügbare Bauland aus, und sie müssten in Reaktion darauf neues Bauland ausweisen oder eine erhebliche Verteuerung und Verdrängung akzeptieren. 


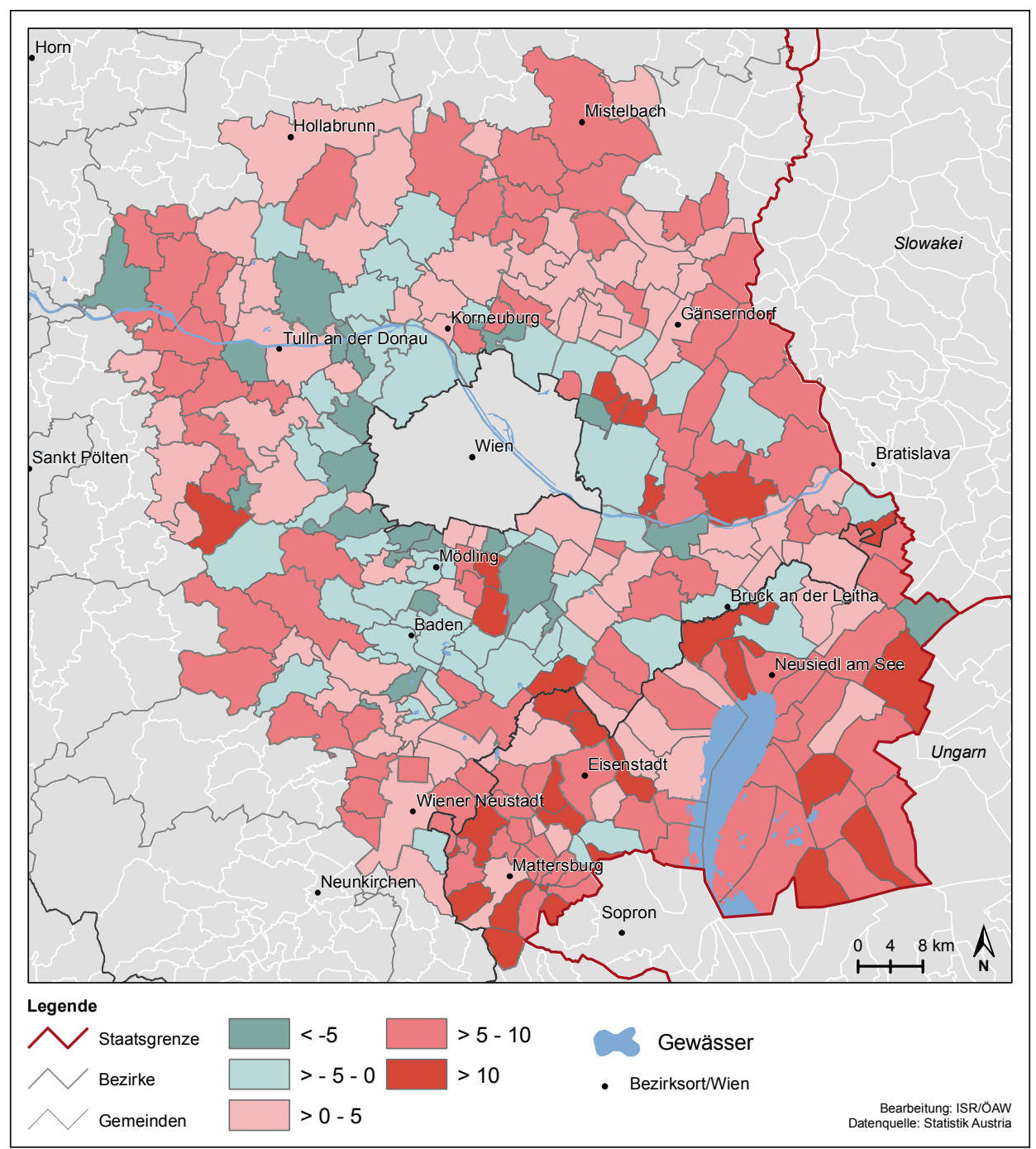

Abb. 2: Restreserve Bauland 2020 (Status-Quo-Szenario 2)

Die geringere Restreserve an verfügbarem Bauland dokumentiert die Zunahme der blau markierten Gemeinden in Abbildung 2. Negative Baulandreserven finden sich im Süden von Wien, in den Politischen Bezirken Baden und Mödling sowie im Politischen Bezirk Wien-Umgebung, aber auch in den Gemeinden im Osten und Norden. Das engere Stadtumland gelangt an seine Wachstumsgrenzen. Aber auch das mosaikartige Muster an „blauen“ Gemeinden findet sich wieder. Dagegen verfügen die Gemeinden im nördlichen und mittleren Burgenland weiterhin über große Wachstumspotenziale. Szenario SQ2 verweist damit abermals auf die Notwendigkeit einer regional gesteuerten Suburbanisierung. 


\section{Bürgermeisterszenario}

Das zweite Szenario ist das sogenannte „Bürgermeisterszenario“, welches im Wesentlichen auf den Aussagen der kommunalpolitisch Verantwortlichen basiert. Dabei zeigt sich, dass von den befragten Gemeinden 97,3\% wachsen wollen. Bei den wenigen Gemeinden, die für das Jahr 2020 eine niedrigere gewünschte Einwohnerzahl angegeben hatten als die gegenwärtige, besteht zumeist entweder der Verdacht, dass die Frage nicht richtig verstanden oder die Angaben fehlerhaft verarbeitet wurden. Nur in sehr wenigen Fällen dürfen Gemeinden aus gut nachvollziehbaren Gründen (z.B. Flughafennähe) nicht wachsen. Insgesamt soll, wenn man den Angaben der Gemeindeverantwortlichen folgt, das Stadtumland nicht 909.000 Einwohner beherbergen, sondern 1,122.000 und damit um rund 213.000 über der Einwohnerzahl des Jahres 2008 liegen. Diese Zahl ist in etwa doppelt so groß wie das von Statistik Austria prognostizierte Wachstum für ganz Niederösterreich. Suburbansierung ist nicht nur, aber auch einer kommunalpolitischen „Self fulfilling prophecy“ zuzuschreiben. Es sind die Gemeindeverantwortlichen, die durch ihre Absicht und ihr raumordnerisches Handeln für eine weiterführende Suburbanisierung sorgen.

Wenn das von den Gemeindeverantwortlichen angegebene Wachstum tatsächlich realisiert wird, überschreiten 67 Gemeinden ihre Baulandreserven, wobei unterstellt wird, dass die gesamte Reserve mobilisiert werden kann. Das ist aber nicht realistisch. Die Gemeindeverantwortlichen selbst haben ja angegeben, dass nur rund ein Drittel des Baulandes tatsächlich mobilisierbar ist. Wenn der Berechnung dieser Wert zugrunde gelegt wird, dann überschreiten bereits 177 Gemeinden ihre Baulandreserven und müssten, um ihr gewünschtes Wachstum zu erreichen, neues Bauland aufschließen und entsprechend widmen. Diese Entwicklung wäre angesichts der hohen, aber eben nicht mobilisierbaren Baulandreserven nicht unproblematisch.

In Abbildung 3 wird auch für diese Annahme die Restreserve kartographisch dargestellt. Das Kartenbild hat sich im Vergleich zum Szenario SQ1/SQ2 deutlich verändert. Die blau markierten Gemeinden mit aufgebrauchten Baulandreserven dominieren. Besonders häufig werden die Reserven im unmittelbaren Stadtumland aufgebraucht sein, aber auch im südlichen Weinviertel und sogar im nördlichen Burgenland. Einzelne „Inseln“ mit noch vorhandenen Baulandreserven werden nur noch im südlichen Wiener Becken, im Seewinkel und in einigen Gemeinden des äußeren Randes der Stadtregion zu finden sein.

Das Bürgermeisterszenario kennzeichnet somit eine extreme Zukunftsvorstellung: Wenn Baulandwidmung und Wachstumsambition ausschließlich in den Händen der Kommunalpolitik verbleiben und ohne Kontrolle umgesetzt werden, dann ist eine weitere „Zersiedlung“ des Umlandes die fast zwangsläufige Folge. Legt man den Bewertungen die Prinzipien eines ökologisch nachhaltigen Wachstums zugrunde, dann markiert das Bürgermeisterszenario wohl die am wenigsten anzustrebende Zukunft. Unsere Schlussfolgerung daraus ist eindeutig: Die Entwicklung des Stadtumlands darf und soll nicht den bottom-up-Prozessen allein überlassen bleiben, denn ein unkoordiniertes Wachstum wäre die Folge. Ein Interventionsszenario ist notwendig. 


\section{Interventionsszenarien}

Die den Interventionsszenarien zugrunde liegende Idee ist relativ einfach, ihre Realisierung jedoch komplex. In den Interventionsszenarien werden keine Trends fortgeschrieben oder Begehrlichkeiten realisiert, sondern der suburbane Raum wird aufgrund planerischer Prämissen maximal aufgefüllt. Als planerische Norm gilt dabei, die Besiedlung dort zu konzentrieren, wo die Ausstattung mit zentralörtlichen Gütern und Diensten für die Bewohner gegeben oder über kurze Wegstrecken zu erreichen ist. Kompakte und verkehrsminimierende Siedlungen sind das Ziel.

\subsection{Erreichbarkeit und Ausstattungsqualität}

Als erstes ist die „Eignung“ einer räumlichen Einheit zur Besiedlung zu bestimmen. Diese Eignung ergibt sich in erster Linie aus der Ausstattung mit und der Erreichbarkeit von Anbietern ausgewählter zentralörtlicher Güter und Dienste über den motorisierten Individualverkehr und den öffentlichen Personennahverkehr. Eine räumliche Einheit ist dann gut zur Besiedlung geeignet, je geringer die Distanz ist, die zurückgelegt werden muss, um Anbieter von Gütern und Diensten zu erreichen. In dieser räumlichen Einheit soll Verdichtung stattfinden, während jene Teile des suburbanen Raums, die schlecht erreichbar sind und über keine gute Ausstattung verfügen, freizuhalten sind. Wenn nämlich dort Bevölkerung angesiedelt wird, dann wäre eine nachträgliche und finanziell aufwändige Erschließung notwendig.

Um die „Eignung“ einer räumlichen Einheit zu bestimmen, wurde das verbaute Siedlungsgebiet einer Gemeinde in Quadratrasterzellen mit 1.500 m Seitenlänge eingeteilt. Für jede Rasterzelle wurde ermittelt, ob bestimmte Ausstattungselemente vorhanden sind oder ob diese Ausstattungselemente in benachbarten Rasterzellen zu finden sind. Wie viele Geschäfte mit Vollsortiment im Bereich des Einzelhandels um ein Beispiel zu geben - sind innerhalb von 10 Minuten Fahrzeit mit dem PKW von einer Rasterzelle aus zu erreichen? Dabei wird auch angenommen, dass das erste und zweite Geschäft mit Vollsortiment zur Ausstattungsqualität einer Rasterzelle ungebrochen beiträgt, die nächsten Geschäfte aber nur noch mit einer abnehmenden Wertigkeit. Dabei spielt es keine Rolle, ob eine Gemeindegrenze überschritten werden muss oder nicht.

Mit der Berechnung der Ausstattungsqualität und der Erreichbarkeit der Rasterzellen wurde das Unternehmen „LOB iC“ vom Amt der Niederösterreichischen Landesregierung (RU2) im Wege der PGO beauftragt (vgl. LANGTHALER 2009). Der untersuchte Raum umfasste die vom Auftraggeber angegebene PGO-Stadtregion. Um Erreichbarkeitspotenziale errechnen zu können, wurde des Weiteren ein „Saum“ um den inneren Raum festgelegt und berücksichtigt. Die Berechnung erfolgte für den beschriebenen Raster, der mit dem vom Amt der Niederösterreichischen Landesregierung verwendeten Erreichbarkeitsraster deckungsgleich ist.

Ziel der Berechnung war das Ermitteln der Anzahl der in einer bestimmten Zeitdistanz von einer Rasterzelle aus zu erreichenden Einrichtungen, um Güter des täglichen und mittelfristigen Bedarfs sowie bestimmte Dienstleistungen konsumieren zu 


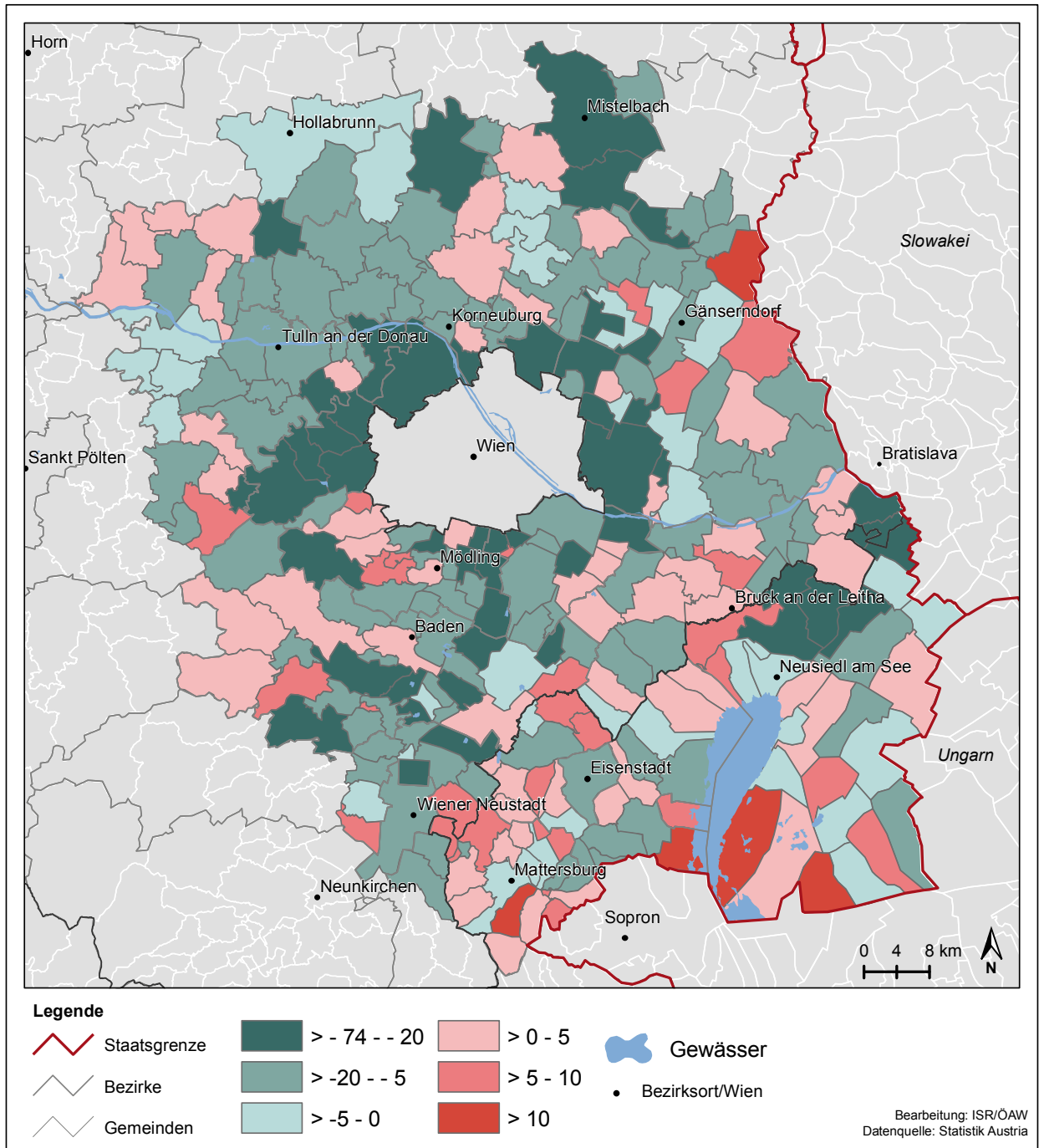

Abb. 3: Restreserve Bauland 2020 (Bürgermeisterszenario)

können. Ist eine Rasterzelle selbst mit diesen Einrichtungen ausgestattet, erhöht sich die Zahl der innerhalb einer vorgegebenen Zeitdistanz erreichbaren Einrichtungen. Ebenso wurden die Indikatoren der Verkehrsanbindung berücksichtigt. Je besser eine Rasterzelle mit Haltestellen des öffentlichen Verkehrs ausgestattet ist oder je besser das nächste Zentrum von einer Rasterzelle aus zu erreichen ist, umso größer ist das Erreichbarkeitspotenzial der Zelle. Rasterzellen mit guter Ausstattung und hohem Erreichbarkeitspotenzial werden als prädestiniert angesehen, zusätzliche Wohnbevölkerung aufzunehmen. Diese Überlegungen spielen bei den Interventionsszenarien eine große Rolle. 


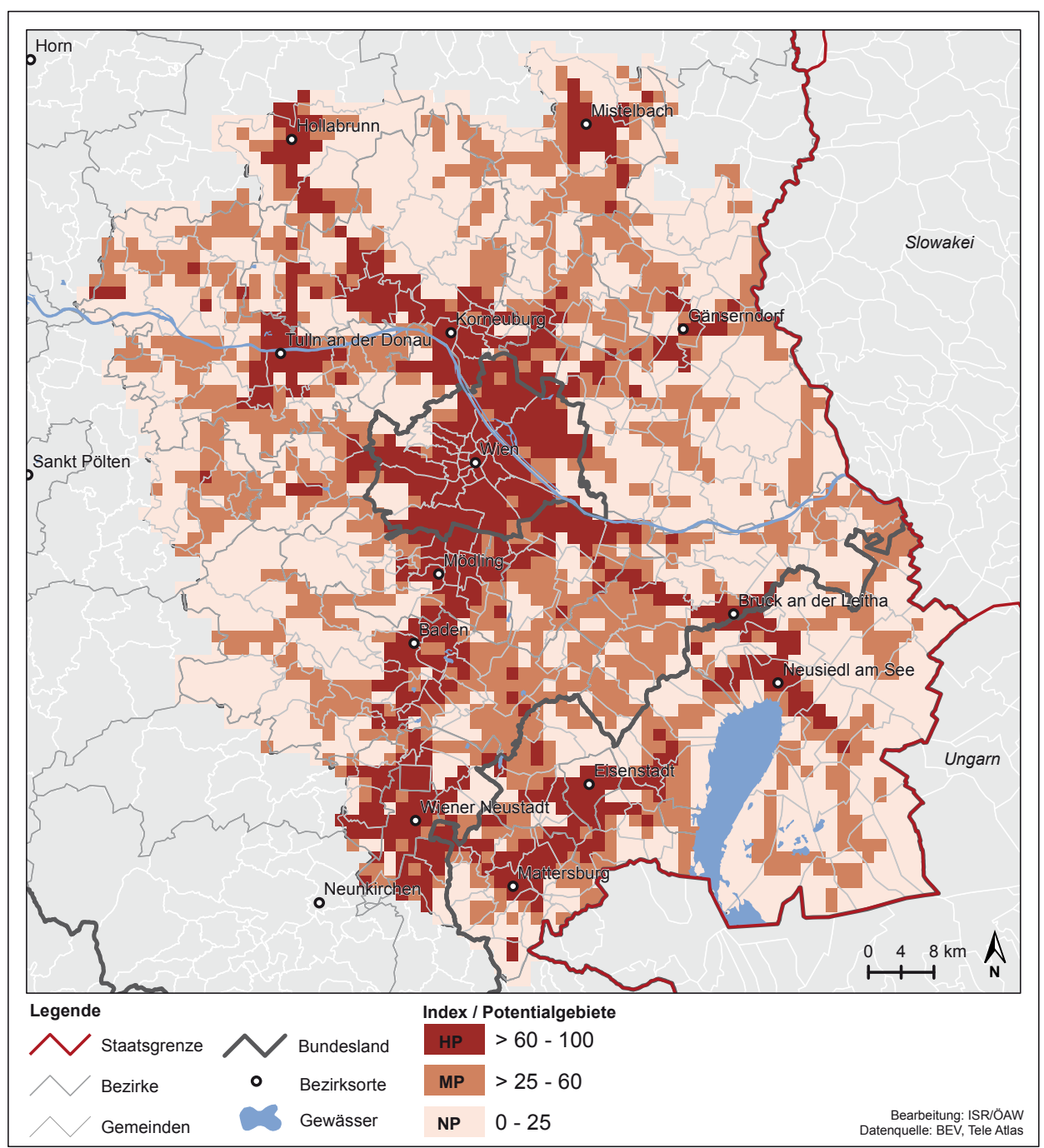

Abb. 4: Erreichbarkeitspotenziale in der SRO

Insgesamt 24 Merkmale wurden zur Ermittlung der Erreichbarkeitspotenziale einer Rasterzelle herangezogen. 13 Merkmale entfallen auf Einrichtungen der Versorgungsinfrastruktur (Nahversorgung, Bildung, Betreuung und Gesundheit) und elf Merkmale auf die Verkehrsanbindung durch den motorisierten Individualverkehr bzw. den öffentlichen Personenverkehr. Die Herold-Branchen-CD sowie ein Erreichbarkeitsmodell bildeten die Grundlage der Berechnung. Die Merkmale der Infrastruktur und der Verkehrsanbindung wurden gewichtet und zusammengezählt. Das Ergebnis ist jedenfalls eine Bewertung der Ausstattungsqualität und der Erreichbarkeitspotenziale der 3.600 Rasterzellen und damit der erwünschten Besiedlung. 
Die Ausstattungsqualität und die Erreichbarkeitspotenziale der 3.600 Rasterzellen wurden am Ende des Verfahrens gegenübergestellt und klassifiziert. Um die Aussage nicht zu kompliziert zu gestalten, wurden lediglich drei Gruppen von Rasterzellen gebildet: (1) Rasterzellen mit sehr guter Ausstattung in den Bereichen Nahversorgung, Bildung, soziale Betreuung und Gesundheit besitzen diese Infrastruktur selbst oder in unmittelbarer Nachbarschaft und werden als Hochpotenzialgebiet bezeichnet; (2) durchschnittlich gut ausgestattete und erreichbare Rasterzellen werden als Mittelpotenzialgebiet bezeichnet; (3) schlecht erreichbare und defizitär ausgestatte Rasterzellen als Niedrigpotenzialgebiet. Die Erreichbarkeit über den öffentlichen Verkehr und den motorisierten Individualverkehr spielt dabei eine große Rolle.

Die kartographische Darstellung dieser drei Gebietstypen reflektiert sehr deutlich die Siedlungs- und Verkehrsstruktur in der Stadtregion (vgl. Abb. 4). Ausstattungsqualität und Erreichbarkeitspotenziale sind durch die vorhandenen Zentren und Achsen geprägt. Tulln an der Donau, Hollabrunn, Mistelbach und Gänserndorf sind die wesentlichen Zentren im Nordwesten, Norden und Nordosten der Stadt Wien. Sie sind partiell durch Schnellstraßen (S3), Eisenbahnen (Westbahn bzw. Nordbahn) oder Schnellbahnen verbunden. Im Südosten fällt die sich entwickelnde Achse entlang der A4 in Richtung Bruck an der Leitha und Neusiedl am See ebenso auf wie die Südachse in Richtung Wiener Neustadt. Eisenstadt, Mattersburg und Wiener Neustadt wiederum bilden ein Städtedreieck mit hohem Erreichbarkeitspotenzial und guter Ausstattung.

\subsection{Empirische Ergebnisse}

Das Wachstum soll - so die Idee des Interventionsszenarios - dort erfolgen, wo dies aufgrund der bestehenden Ausstattung und Erreichbarkeitspotenziale vorteilhaft erscheint. Suburbanisierung soll stärker strukturiert werden und nicht allein von der Verfügbarkeit von Bauflächen geleitet sein. Was durch das Interventionsszenario verhindert werden soll, ist ein unstrukturiertes und zufälliges Auffüllen der bestehenden Baulandreserven. Ohne Interventionen würde die zukünftige Suburbanisierung einem Sprawling entsprechen. Wohnbevölkerung siedelt sich in einem solchen Fall dort an, wo Bauland zu den vergleichsweise günstigsten Preisen zur Verfügung steht, unabhängig davon, ob dieses Bauland aus Gründen der Erreichbarkeit im öffentlichen Personennahverkehr oder der lokalen Infrastruktur besonders geeignet erscheint. Ein mosaikartiges Siedlungsbild entsteht in der Suburbia, welches autoabhängig und ökologisch nachteilig ist.

Werden jedoch jene Siedlungsgebiete zuerst „aufgefüllt“, die dazu prädestiniert sind, dann ergibt sich eine Verteilung, die sich an den bestehenden Achsen, Zentren und Verdichtungsräumen orientiert. Die durch das Interventionsszenario aufgeworfene Frage lautet dabei: Reichen die wenigen und einfachen Regeln hinsichtlich der Verdichtung und der Baulandmobilisierung in Abhängigkeit von den Hoch-, Mittelund Niedrigpotenzialgebieten aus, um das vorhergesehene Wachstum zu bewältigen? 


\begin{tabular}{|l|c|c|c|c|c|c|}
\hline & \multicolumn{2}{|c|}{ ÖV mit 60 gewichtet } & \multicolumn{2}{c|}{ ÖV mit 80 gewichtet } & \multicolumn{2}{c|}{ ÖV mit 20 gewichtet } \\
\hline $\begin{array}{l}\text { Erreichbarkeits- } \\
\text { potenzial }\end{array}$ & $\begin{array}{c}\text { Zusätzliche } \\
\text { Einwohner }\end{array}$ & in \% & $\begin{array}{c}\text { Zusätzliche } \\
\text { Einwohner }\end{array}$ & in \% & $\begin{array}{c}\text { Zusätzliche } \\
\text { Einwohner }\end{array}$ & in \% \\
\hline Niedrig & 19.917 & 11,0 & 21.536 & 13,1 & 18.544 & 9,4 \\
\hline Mittel & 53.790 & 29,7 & 65.307 & 39,7 & 42.775 & 21,8 \\
\hline Hoch & 107.266 & 59,3 & 70.528 & 47,2 & 135.020 & 68,8 \\
\hline Insgesamt & 180.974 & 100,0 & 164.423 & 100,0 & 196.339 & 100,0 \\
\hline
\end{tabular}

Quelle: eigene Berechnungen

Tab. 2: Interventionsszenario: öffentlicher Verkehr (ÖV) unterschiedlich gewichtet

Das Interventionsszenario liefert eine eindeutige Antwort: Werden die Rasterzellen nach den Regeln des Interventionsszenarios aufgefüllt, dann gelangt man zu einer Summe von zusätzlich 181.000 Einwohnern im Stadtumland. Mit anderen Worten: Werden die Baulandreserven in Abhängigkeit von den Erreichbarkeitspotenzialen mobilisiert und unterschiedlich dicht verbaut, dann können insgesamt und zusätzlich 181.000 Menschen untergebracht werden, ohne dass zusätzliches Bauland notwendig wird - und unter Befolgung raumordnerischer Prinzipien. 11\% der zusätzlichen Wohnbevölkerung sind in den Niedrigpotenzialgebieten verteilt worden, $30 \%$ in den Mittelpotenzialgebieten und 57\% in den Hochpotenzialgebieten (vgl. Abb. 5).

In diesem Szenario wird der Einfluss der Verkehrsanbindung mit dem öffentlichen Verkehr (ÖV) mit 60\% gewichtet und jener des Individualverkehrs mit 40\%. Wird dem öffentlichen Verkehr ein höheres Gewicht beigemessen (Gewichtung 80\%), dann sinkt die Zahl einer fiktiven zusätzlichen Wohnbevölkerung auf 164.000 infolge räumlicher Konzentration auf jene Teilräume, die über den öffentlichen Verkehr gut angebunden sind. Schreibt man dagegen dem Individualverkehr ein höheres Gewicht zu (80\%), dann steigt die Zahl an Rasterzellen mit hohem Erreichbarkeitspotenzial und damit auch die zusätzlich mögliche Wohnbevölkerung auf 196.000. Suburbanisierung würde sich bei einem höheren Gewicht des Individualverkehrs flächiger ausbreiten und mehr Menschen könnten sich in Hochpotenzialgebieten ansiedeln.

Damit sind aber auch die Grenzen der zusätzlich aufnehmbaren Wohnbevölkerung abgesteckt, denn die potenziellen Bewohner des Stadtumlands würden wahrscheinlich keine andere Gewichtung des Individualverkehrs und des öffentlichen Verkehrs vornehmen als eine solche zwischen 20 und 80 oder 80 und 20. Ohne zusätzliches Bauland aufzuschließen, aber bei konsequenter Befolgung einfacher planerischer Regeln (Verdichtung in jenen Gebieten, die gut erreichbar sind), können im Stadtumland zwischen 164.000 und 196.000 Menschen zusätzlich aufgenommen werden, jedenfalls deutlich mehr als prognostiziert. 


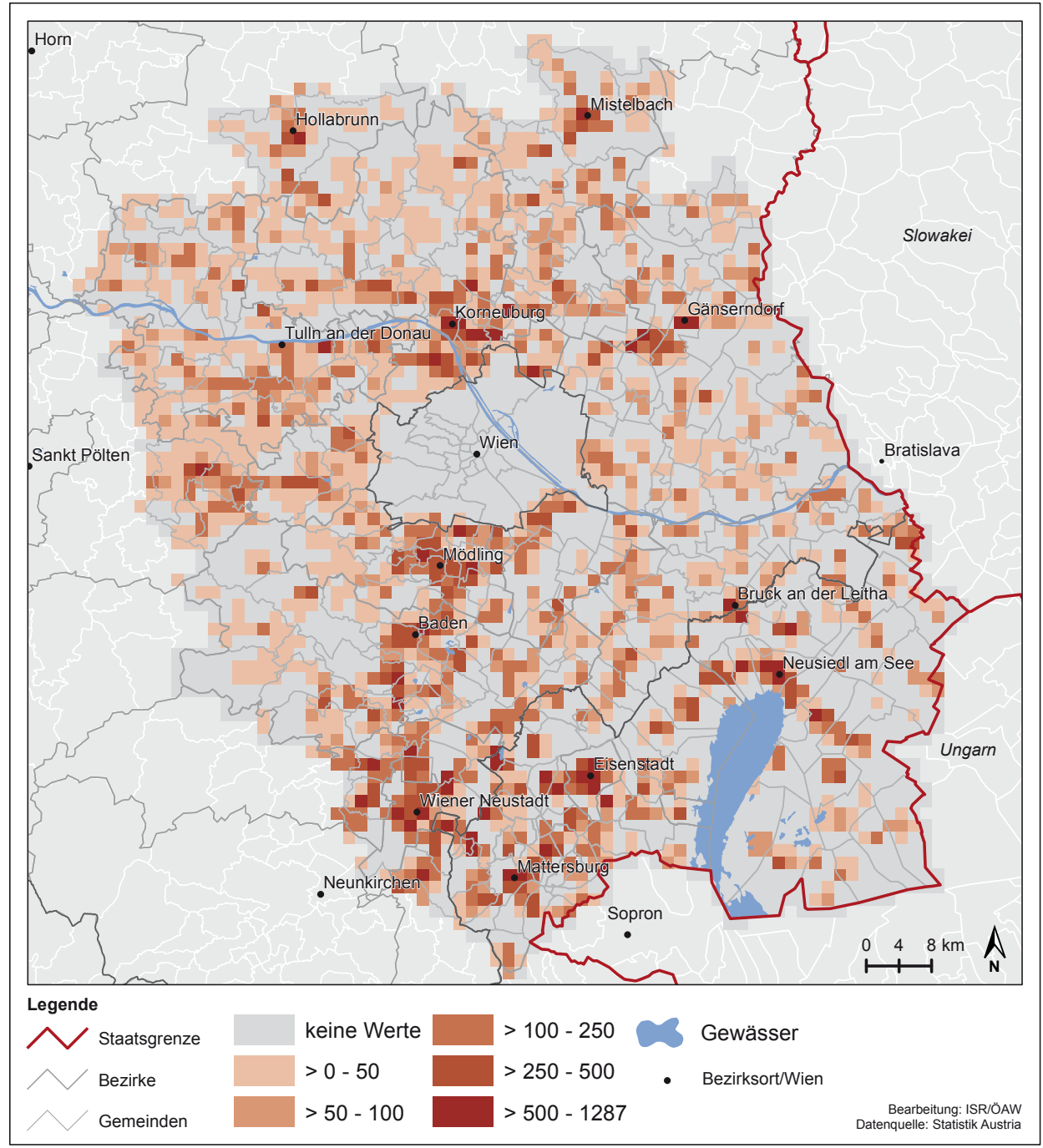

Abb. 5: Zusätzliche Wohnbevölkerung (Interventionsszenario; ÖV-Gewichtung 60\%) 


\section{Fazit}

Die Stadtregion wird wachsen. Alle relevanten Prognosen und Entwicklungen deuten dies an. Sie wird der Einwohnerzahl nach wachsen, weil sowohl die Stadt Wien ein Mehr an Wohnbevölkerung erwartet als auch das Stadtumland. Die Stadt Wien ist als Zuwanderungsziel attraktiv und verzeichnet einen hohen positiven Wanderungssaldo mit dem Ausland. Das Stadtumland ist ebenso attraktiv für jene Menschen, die Wien verlassen, um in einem Eigenheim „,im Grünen“ zu wohnen, aber auch für die Zuwanderer aus anderen Teilen Österreichs, die vielleicht in der Kernstadt arbeiten, aber außerhalb derselben wohnen wollen. Dafür sprechen vergleichsweise geringere Wohnungskosten und die gute Erreichbarkeit (innerhalb) der Stadtregion.

Die Szenarien zeigen aber deutlich auf, dass nur eine Kombination von vollständiger Baulandmobilisierung und abgeschwächtem Wachstumstrend im Stadtumland zu einem Ergebnis führt, bei dem kein neues Bauland aufgeschlossen werden muss (SQ1). Wenn die Mobilisierbarkeit von Bauland eingeschränkt wird, explodiert förmlich die Zahl der Gemeinden, die innerhalb einer Dekade ihr Bauland restlos verbraucht haben und neues benötigen würden. Da hilft auch eine mäßige Verdichtung wenig. Sie kann den Trend nicht aufhalten, es sei denn, die Gemeinden entschlössen sich zu einer deutlich höheren Verdichtung der Wohnbevölkerung.

Noch drastischer wird die Situation, wenn das Wachstum so erfolgt wie es die befragten Gemeindeverantwortlichen in einer Erhebung angegeben haben; insbesondere auch dann, wenn das von ihnen angegebene Ausmaß an Baulandmobilisierung berücksichtigt wird. In diesem Fall würden fast zwei Drittel aller Gemeinden am Ende des Betrachtungszeitraums die Baulandreserven aufgebraucht haben. Der Wunsch nach Umwidmung wäre spätestens dann gegeben und könnte auch schwerlich abgelehnt werden. Die Stadtregion würde sich regellos ausbreiten, auf Erreichbarkeiten würde nicht systematisch geachtet.

Das Szenario der „strukturierten Stadtregion“ belegt schließlich, dass die Anwendung einiger einfacher und einsichtiger Regeln zu einer Entwicklung der Stadtregion führt, die das Wachstum verarbeitet und zudem aus umweltpolitischen und volkswirtschaftlichen Gründen vorteilhaft ist. Es führt zu einer polyzentrischen Entwicklung mit Verkehrsachsen, der Betonung der Stadtnähe und dem Freihalten der Ränder und Zwischenräume. Eine strategisch abgeklärte Entwicklung der wachsenden Stadtregion ist jedenfalls möglich und ratsam - wenn der politische Wille vorhanden ist, regionale Planungsprinzipien über die kommunale Planungspraxis zu stellen und auf deren Einhaltung zu achten. Nicht weniger und nicht mehr Planung ist notwendig, sondern eine, die regional richtig verankert ist. 


\section{Literaturverzeichnis}

Amt der NÖ Landesregierung, Gruppe Raumordnung, Umwelt und Verkehr - Abteilung Raumordnung und Regionalpolitik (Hrsg.) (2004), Strategie Niederösterreich. Landesentwicklungskonzept. St. Pölten.

Bölling L., Sieverts Th. (2004), Mitten am Rand. Auf dem Weg von der Vorstadt üder die Zwischenstadt zur regionalen Stadtlandschaft (= Schriftenreihe Zwischenstadt, 1). Wuppertal.

Brake K., Einacker I., Mäding H. (2005), Kräfte, Prozesse, Akteure - zur Empirie der Zwischenstadt (= Schriftenreihe Zwischenstadt, 3). Wuppertal.

Bundesamt Für Bauwesen und Raumordnung (BBR) (Hrsg.) (2007), Akteure, Beweggründe, Triebkräfte der Suburbanisierung. Motive des Wegzugs - Einfluss der Verkehrsinfrastruktur auf Ansiedlungs- und Mobilitätsverhalten. - http://www.bbsr.bund.de/cln_015/ nn_622162/BBSR/DE/Veroeffentlichungen/BBSROnline/2007/DL_ON212007,templateId= raw, property = pubicationsFile.pdf/DL_ON212007.pdf (letzter Zugriff 21/2007)

Fassmann H., Görgl P., Helbich M. (2009), Atlas der wachsenden Stadtregion. Materialienband zum Modul 1 des Projekts „Strategien zur räumlichen Entwicklung der Ostregion (SRO)“. Wien.

Görgl P. (2008), Die Amerikanisierung der Wiener Suburbia? Der Wohnpark Fontana. Eine sozialgeographische Studie. Wiesbaden, VS-Verlag.

Helbich M. (2009), Modellierung (post)suburbaner Prozesse am Fallbeispiel der Stadtregion Wien. Wien - Berlin, LIT-Verlag.

ILS - INSTITUT FÜR LANDES- UND STADTENTWICKLUNGSFORSCHUNG (Hrsg.) (2008), trends - Wohnstandortentscheidungen in der Stadtregion: Das Beispiel „Bergisches Land“. - http:// www.ils-forschung.de/down/ils_trends_2_08.pdf (letzter Zugriff 02/2008)

IMU - Institut FÜr Medienforschung Und URbanistik (Hrsg.) (2002), Raus aus der Stadt? Untersuchung der Motive von Fortzügen aus München in das Umland 1998-2000. München [im Auftrag des Referats für Stadtplanung und Bauordnung der LH München].

Koop Th. (2007), Demografischer Wandel und Wohnungsmarktentwicklung. Auswirkungen, Einflüsse und Prognosen. Saarbrücken, VDM.

Langthaler Th. (2009), WAQ - Werkzeug Ausstattungsqualität. Mimeographiertes Manuskript, Wien.

LoibL W. et al. (Hrsg.) (2002), „STAU-Wien“ Stadt-Umlandbeziehungen in der Region Wien: Siedlungsentwicklung, Interaktionen und Stoffflüsse, Endbericht - Teil A“" (= ARC Seibersdorf research Report). Seibersdorf.

Musil R., PINDuR P. (2008), Nachhaltige Suburbanisierung? Entwicklungstrends und Steuerungsmechanismen der Siedlungstätigkeit im Biosphärenpark Wienerwald. Projektendbericht zum MAB-Forschungsprojekt „Nachhaltige Suburbanisierung“. Wien.

Österreichisches Institut Für ERWachSEnEnbildung (ÖIEB) (Hrsg.) (2004), Motivation und Zufriedenheit von Zuzüglern ins Wiener Umland. Zusammenfassung, Resümee, Empfehlungen. St. Pölten.

ÖSTERREICHISCHE RAUMORDNUNGSKONFERENZ (ÖROK) (Hrsg.) (2009), Räumliche Entwicklungen in österreichischen Stadtregionen. Handlungsbedarf und Steuerungsmöglichkeiten. Wien. 